\title{
Exploring the limits of safety analysis in complex technological systems
}

\author{
D. Sornette* \\ Department of Management, Technology and Economics, \\ ETH Zurich, Scheuchzerstrasse 7, CH-8092 Zurich, Switzerland \\ T. Maillart列 \\ Department of Humanities, Social and Political Sciences, \\ ETH Zurich, Ramistrasse 101, CH-8092 Zurich, Switzerland \\ W. Krögett \\ Risk Center, ETH Zurich, Scheuchzerstrasse 7, CH-8092 Zurich, Switzerland
}

(Dated: May 21, 2018)

From biotechnology to cyber-risks, most extreme technological risks cannot be reliably estimated from historical statistics. Therefore, engineers resort to predictive methods, such as fault/event trees in the framework of probabilistic safety assessment (PSA), which consists in developing models to identify triggering events, potential accident scenarios, and estimate their severity and frequency. However, even the best safety analysis struggles to account for evolving risks resulting from inter-connected networks and cascade effects. Taking nuclear risks as an example, the predicted plant-specific distribution of losses is found to be significantly underestimated when compared with available empirical records. Using a novel database of 99 events with losses larger than $\$ 50 ’ 000$ constructed by Sovacool, we document a robust power law distribution with tail exponent $\mu \approx 0.7$. A simple cascade model suggests that the classification of the different possible safety regimes is intrinsically unstable in the presence of cascades. Additional continuous development and validation, making the best use of the experienced realized incidents, near misses and accidents, is urgently needed to address the existing known limitations of PSA when aiming at the estimation of total risks. 


\section{INTRODUCTION}

Most innovations are adopted on the premise that the upside gains largely make up for the downside short- and long-term risks, in particular through the adoption of safety measures aiming at preventing or mitigating potential losses. But innovations are often disruptive and, by essence, break new ground. This implies that history is a poor guide for risk assessment due to the novelty of the technology and the corresponding insufficient statistics. For highly technical enterprises for which full scale experiments are beyond reach (such as the Internet and smart grid technologies and associated cyber-risks, technological and population growth and climate change, financial innovation and globalization and the dangers of systemic banking crises), engineers resort to simulation techniques and scenariobased analyses.

To be concrete, we restrict our discussion to the nuclear industry, which has been a leader in the development of state-of-the-art safety analysis, with outstanding efforts aimed at preventing incidents from becoming major accidents. For this, probabilistic safety assessment (PSA) has been developed as a decision support tool aiming at ensuring a high level of plant safety limiting the risks of possible release of radioactivity. PSA consists in developing fault and event tree models to simulate accidents, their different triggers and induced scenarios, their severities as well as their estimated frequency [1, 2]. When performed as an ongoing process continuously refined using the information of new examples, plant-specific safety analysis has proved very useful for the implementation of ever better safety barriers, keeping the advantages of the technology while reducing its undesirable dangers. PSA is a well-established discipline with growing applications in support of rational decision-making involving important technological and societal risks.

The article is organized as follows. Section 2 presents a brief description of the PSA methodology. Section 3 describes the main predictions of PSA with respect to the plant specific "core damage frequencies" and the "large early release frequencies", before comparing them quantitatively with the database of losses per event constructed by Sovacool [18], with special care to demonstrate the robustness of the reported power law distribution.

\footnotetext{
*Electronic address: dsornette@ethz.ch

†Electronic address: tmaillart@ethz.ch

${ }^{\ddagger}$ Electronic address: kroeger@mavt.ethz.ch
} 
Section 4 introduces and analyzes a simple conceptual model of cascades of failures that allows us to rationalize the discrepancy between predicted and realized losses. Section 5 concludes.

\section{BRIEF DESCRIPTION OF PROBABILISTIC SAFETY ASSESSMENT (PSA)}

PSA provides nuclear plant-specific information on risk metrics at three sequential levels of end states (levels 1-3). Level 1 corresponds to the assessment of the risk of a core damage (core damage frequency or CDF). Level 2 assesses the size of radioactive releases from the reactor building, in the event of an accident (large early release frequency or LERF), in order to develop accident management strategies and identify potential design weaknesses in reactor containment buildings. Core damage frequency (level 1) and large early release frequency (level 2) are regarded as representative surrogates to steer design and operations of systems towards achieving quantitative and qualitative safety goals. Level 3 evaluates the impact of such releases on the public and the environment and is used mainly for emergency planning. In the nuclear domain, PSA Levels 1 and 2 are required to support regulatory activities in most countries (e.g., in the US since 1995 to complement the deterministic approach within the framework for risk informed regulation). The PSA methodology has developed into national and international guidelines (for an history of PSA, see for instance chapter 2.4 in Ref. [3] and chapter II-b in Ref. [4]). International guidelines are presented in Refs. [5, 6] and Ref. [7] (for the later, see http://www-ns.iaea.org/standards/ for updates and revisions). This has resulted in widely established and used codes, such as SAPHIRE 8 (Systems Analysis Programs for Hands-on Integrated Reliability Evaluations), MELCOR (see for instance Ref. [8]) and MACCS2 (MELCOR Accident Consequence Code System, Version 2).

Since many years, PSA is not thought to represent the true risks and to become generalized across sectors, countries and events without considerable adjustments. PSA is mainly thought of as a platform for technical exchanges on safety matters between regulators and the industry, among peers, between designers and operators [9]. PSA provides a rigorous and methodical way to steer towards measures to achieve safety goals by efficient use of resources and measures. PSA is a fairly well developed mature methodology, although diversely implemented and used in different countries, in particular in the choice of internal 
and external events that are included in the analysis.

The basic methodology of PSA is based on logic trees and human reliability analysis (HRA) incorporating unintentional failures only, with the uncertainty analysis being restricted to data variation that assume traditional (Normal) distributions. Common cause failure $(\mathrm{CCF})$ analysis is included by point estimates on fractions of the system, with rare event approximations and cut-sets with cut-off values for the quantification of fault trees and binning techniques to cope with the large number of sequences. PSA uses in general the assumption that a nuclear power plant is essentially a closed system, even under catastrophic accident conditions. There are considerable weaknesses concerning the neglect of cascades and the transformation of PSA modeling to consider the nuclear power plan as an open system with strong interactions with its environment as well as to include the importance of local conditions, in particular under severe accident conditions. PSA is limited to single units embedded in an ideal environment, in which a safety culture is assumed to be ensured, and for which atmospheric transport and dispersion dominates (thus neglecting other pathways). This has implications for the conclusions, when the nature of the triggering event (earthquakes, flooding / tsunamis) affect large areas, and/or other sources of radiation (spent fuel pool) exist, which are currently neglected. There is also limited feed of accident insights back into PSA methodology as well as few new approaches and models developed in other sectors and fields that influence PSA.

\section{CONFRONTING PSA WITH REALITY}

\section{A. Understanding the predictions of PSA}

Probabilistic safety assessment is seldom used for communication but, in reality, it is sometimes used to support political decisions, within the public debate and by actors other than the nuclear industry such as the insurance industry. And this naturally raises the issue of the possible gaps between the occurrence of accidents, public perceptions and the predictions of PSA.

In order to frame correctly the discussion on how to use PSA to estimate risk surrogates such as core damage frequency (CDF) and large early release frequency (LERF), we stress again that the basic methods of PSA levels 1 and 2 use fault/event trees, human reliability 
analysis (HRA) and common cause failure (CCF), to model plant and operator behavior according to principles of reductionism in a plant specific way limited to single units. In this way, PSA models failure of safety systems using linear semi-dynamic causal chains, with binning techniques and many approximations allowing quantification. And severe accident management guidelines (SAMG) are taken into account in such framework, as long as they are planned and trained.

PSA level 3 studies are relatively rare, and uses of PSA to estimate risks for the public are mostly based on levels 1 and 2 studies, which make assumptions about population behavior, take deterministic (early) and stochastic (late cancer effects) into account for submersion, inhalation and ingestion exposure pathways. They are site-specific but extend the calculations of consequences to large distances $(800 \mathrm{~km})$ to capture low dose latent effects. "Old" studies include Refs. [10, 11, 20], which have been criticized for instance by Marshall [12] and Speed [13]. Revisions of "old" studies include Ref. [14], NUREG-1150 [15] and NEEDS [16], the later uses generic, simplified tree techniques for EPR at sites in five countries.

Plant specific core damage frequencies $(\mathrm{CDFs})$ for light water reactors obtained by PSA vary between $10^{-4} /$ reactor-year and $10^{-5}$ /reactor-year (chapter 2.5.2 in Ref. [3]) and go down to $10^{-6} /$ reactor-year for newly built plants (EPR in Finland have CDF of $2 \cdot 10^{-6} /$ reactor-year) or well back-fitted plant (such as the KKL plant in Switzerland). These values are accepted by licensing authorities after thorough review. Compared with these general CDFs values of $10^{-4}-10^{-5}$ /reactor-year, plant specific LERFs demonstrating containment capabilities are typically one order of magnitude lower, in the range $10^{-5}-10^{-6} /$ reactor-year.

Concerning fatalities, the above cited studies show maximum consequences of about $10^{5}$ latent fatalities at a probability of $10^{-8}$ per reactor-year or even lower frequency level and steep decrease as improvements are implemented (see e.g. chapter 2.5.3 of Ref. [3] and Ref. (4]). These high consequence numbers are due to an assumed linear dose-risk relationship.

\section{B. Comparison between PSA frequencies and empirical data}

Over the human history of nuclear power, there has been 3 to 5 core damage events for a total of about 10'000-15'000 reactor-years, depending on whether the three core damage 
of three of the five reactors in the Fukushima Dai-ichi nuclear plant in Japan are counted as 1 or 3: (i) the Three Mile Island accident (TMI) in the US on March 28, 1979, (ii) the Chernobyl nuclear accident in Ukraine (former Soviet Union) on 26 April 1986 and (iii) the Fukushima Dai-ichi nuclear disaster following the Tohoku earthquake and tsunami on 11 March 2011.

Using the most conservative figure of $10^{-4}$ /reactor-year for CDFs for light water reactor, one would thus expect typically only 1 such event over the 10'000-15'000 reactor-years of the human nuclear plant history. More striking, using the most conservative figure for LERF of $10^{-5}$ /reactor-year, one would expect 0.1 such event over the course of human history and not the 2-4 large releases of radioactive elements in the environment (here, the Three Mile Island accident is not counted as the release of radioactive gases and radioactive iodine was small). These discrepancies are worrisome but they deal with small numbers for which statistics is by definition unreliable and therefore broad conclusions are unreliable.

To advance the discussion, we propose to enrich the statistics by using complementary metrics, here the complementary cumulative distribution function (CCDF) (also known as survival distribution) of the losses $S$ per event (accidents and incidents). Here, one must be clear about the terminology. The US Nuclear Regulatory Commission (NRC) classifies "incidents" are unforeseen events and technical failures during normal plant operation with no offsite release of radioactive elements or severe damage of equipment. In contrast, "accidents" usually refer to offsite release of radiation and/or damage to plant equipment. In the seven-level system of the International Nuclear and Radiological Event Scale, levels 1-3 are "incidents" and level 4-7 are "accidents." The TMI accident was rated level 5 while both Chernobyl and Fukushima Dai-ichi accidents are level 7 and these events are the only reported accidents of nuclear plants over the human history of civil use of nuclear power.

Because these accidents concern only the extreme tail of the distribution, a statistically sound picture can only emerge by extending the analysis to the body of the distribution. The goal is to populate the distribution in the region of large and medium size events, to complement the paucity of statistics on the extreme tail of the distribution, the one associated with the three major accidents mentioned above. For this, we use the database constructed by Sovacool [18], who extends the definition usually taken by the nuclear industry and regulators to include incidents that either resulted in the loss of human life or more than $\$ 50,000$ in property damage. This database includes 99 nuclear events worldwide from 1952 to 2009 
that occurred in different kinds of nuclear facilities. The database has been constructed by searching historical archives, newspaper and magazine articles, and press wire reports, leading to a database of 99 incidents with an estimation of damage they generated, including loss of production and property damage [18]. These nuclear incidents include hydrogen explosion that damage reactor interior, fuel rod catching fire and contaminating facility, partial core meltdown, cooling system malfunctions, steam generator leaks, fire damage of control cables leading to disabling core cooling systems, electrical error causing fire destroying control lines and main coolant pumps, mechanical failure during fuel loading causing corrosion of reactor and release of radioactivity into the plant area, failed tube bundles in steam generators, damaged thermal shield and core barrel support, damage to recirculation system pipeline, instrumentation systems malfunction during startup, corroded reactor coolant pumps and shafts leading to reactor shutdowns, and so on.

Figure 1 shows a one-to-one comparison of the CCDF of damage respectively generated from PSA simulations and from the database of the 99 nuclear events constructed by Sovacool [18]. For the PSA simulations, we refer to the so-called Farmer curve of the 1950s, superseded by the Rasmussen curve of the 1970s [17, 20] for 100 reactors, which is basically confirmed by others (German Risk Study, figures 8-13 of Ref. [10]) or NUREG [15]. It is appreciated by specialists in charge with PSA that these curves may well underestimate extreme events. For our purpose to advance the discussion on an evidence based platform, the PSA curve predicts a rather thin tail while the empirical distribution of losses is fat-tailed, a power law

$$
\operatorname{Pr}(\operatorname{loss} \geq S)=C / S^{\mu}
$$

with an exponent $\mu \approx 0.7$. Concretely, this means that, for nuclear power events with damage costing more than one billion dollars, their frequencies are underestimated by about two orders of magnitude. Moreover, rather than being associated with just a few extreme cases, the existence of a single power law to quantify the distribution of losses over wildly different magnitudes suggests that the problem has intrinsic structural roots.

\section{Robustness of Empirical Distribution}

To assess how much to trust the empirical power law distribution of losses reported in figure 1, we assess its robustness over time. Indeed, an obvious concern with respect to 
the analysis presented in Figure 1 is that we are combining events involving different types of nuclear facilities and of plants, in particular, with different technologies and generations as well as of varying operational contexts. But nuclear plants and their safety procedures are continuously updated, resulting in major technological improvements over time [19]. This suggests that the distribution of losses should change to reflect that extreme risks are becoming less and less probable. Indeed, following the TMI (1979) and Chernobyl (1986) accidents, it is a fact that safety has improved. The release of the WASH-1400 Report in the United States has consecrated the adoption of PSA compared with more simplistic safety methods, followed by widespread adoption by other countries [20].

Figure 2 tests empirically these points. Panel (A) shows the cumulative number of significant events over time from Ref. [18]. Three regimes can clearly be distinguished. In particular, following the Chernobyl's accident, the rate of incidents has been reduced roughly by $70 \%$, most likely as a result of additional safety measures worldwide [19]. Panel (B) of figure 2 shows the three empirical distributions of damage in each of these three periods (first period ending at the TMI accident, second period until the Chernobyl accident and third period ending in 2011), together with the distribution over the whole period from 1952 to 2011. It is remarkable that no statistically significant difference can be found, notwithstanding the very different incident rates shown in panel (A) of figure 2. This suggests that, while safety improvements of nuclear plants and installations had very positive effects preventing the initiation of events, therefore reducing their occurrence rate, no significant change in the structure of the tail distribution (i.e. of the relative likelihood of extreme risks compared with small risks) can be observed. This suggests that improved safety procedures and new technology have been quite successful in preventing incidents (as well as accidents), but mitigation has not significantly improved. This is a paradox, since safety measures should also be designed to minimize the likelihood that, following initiation, incidents worsen and breach one or several of the seven defense-in-depth safety barriers that protect the infrastructure of a typical nuclear plant. Safety barriers include: (i) prevention of deviation from normal operation, (ii) control of abnormal operation, (iii) control of accidents in design basis, (iv) internal accident management including confinement protection and (v) off-site emergency response [22].

As further tests of the reliability and robustness of the power law description, the right panel of Figure 3 shows the survival distribution functions of loss per nuclear incident, broken 
down in periods of times from 1957 to 2011 with 25 events in each time interval. The tails of the distributions for the five time periods have the same power law structure, as confirmed by the maximum likelihood estimates (MLE) of the tail exponent $\mu$ for each distribution shown in the right panel.

\section{CONCEPTUAL MODEL OF CASCADES OF FAILURES}

\section{A. Motivations}

In order to rationalize this discrepancy between predicted and realized losses, we propose a simple conceptual model that embodies the observation that most important industrial accidents involve cascades with inter-dependent and mutually amplifying effects [23]. As reviewed above, such cascades are usually modeled in probabilistic safety assessment by using fault and event tree techniques [17]. We suggest that the discrepancy discussed in subsection IIIB may results from the complexity of the nonlinear cascades that is likely to obfuscate some critical paths that turns out to dominate the generation extreme events. In other words, we suggest that simple models may have a role to complement more complex models. For instance, simple time-dependent statistical models running on desktops may over-perform large-scale general circulation models requiring super-computers for the prediction of climate cycles such as the Southern Oscillation Index and El Niño event [24]. The prediction of material failure has been shown to be feasible and successful with simple models capturing the essence of the mechanism of positive feedbacks during the damage process [25, 26], an approach that can be extended to other complex dynamical systems [27]. Similarly, complex intermittent dynamics can be quite well represented by simple Boolean delay equations [28]. Moreover, it has recently been shown that combining simple and complex models can improve predictive accuracy and quantify better predictive uncertainty, with applications to environmental and climate science [29].

In this spirit, we consider a simple model of cascades of failures, in which an accident is modeled as a succession of unbroken and broken safety barriers with increasing damage. Our goal is to use the simple conceptual model to translate the discrepancy shown in Figure 1 into technically meaningful insights. 


\section{B. Definition and main properties}

Starting from an initiating event generating a damage $S_{0}$, we assume that an event may cascade into a next level with probability $\beta$ with associated additional damage $\Lambda_{1} \cdot S_{0}$. When this occurs, the cascade may continue to the next level, again with probability $\beta$ and further additional damage $\Lambda_{2} \Lambda_{1} \cdot S_{0}$. The probability for the incident to stop after $n$ steps is $P(n)=\beta^{n}(1-\beta)$. After $n$ steps, the total damage is the sum of the damage at each level:

$$
S_{n}=S_{0} \sum_{k=1}^{n} \Lambda_{1} \ldots \Lambda_{k} .
$$

Thus, $S_{n}$ is the recurrence solution of the Kesten map [30, 31]:

$$
S_{n}=\Lambda_{n} S_{n-1}+S_{0}
$$

As soon as amplification occurs (technically, some of the factors $\Lambda_{k}$ are larger than 1), the distribution of losses is a power law, whose exponent $\mu$ is the function of $\beta$ and of the distribution of the factors $\Lambda_{k}$ that solves the equation $\mathrm{E}\left[\Lambda_{k}^{\mu}\right]=1$ [30 32$]$.

The multiplicative mature of the damage represented in expression (2) captures a coarsegrained modeling of the law of "proportional growth" such that future damages are proportional to present damage, for instance via a branching process. Alternatively, one can view the multiplicative structure as reflecting a sequence of triggering events, the larger the existing damage, the larger is the future potential future damage that can be triggered by it. This proportional growth of damage is not thought to represent faithfully the details of each damage path but rather embodies a coarse-grained average law. In a sense, future damage events "preferentially attachment", result from or are triggered via various pathways by existing damage.

In the case where all factors are equal to $\Lambda$, this model predicts three possible regimes for the distribution of damage: thinner than exponential for $\Lambda<1$, exponential for $\Lambda=1$, and power law for $\Lambda>1$ with exponent $\mu=|\ln \beta| / \ln \Lambda$, as shown in the next subsection.

\section{Solution of the cascade model for homogenous factors $\Lambda_{k}=\Lambda$}

The following is a detailed study of the possible behaviors of the model, in particular the different regimes around the critical point $\Lambda=1$.

Three regimes must be considered: 
1. For $\Lambda<1$, the distribution is given by

$$
P_{\Lambda<1}(S \geq s)=(1-\beta)\left(1-\frac{s}{s_{\max }}\right)^{c}, \quad s_{\max }:=\frac{S_{0} \Lambda}{1-\Lambda}, \quad c:=\frac{\ln \beta}{\ln \Lambda}>0 .
$$

This distribution can be approximated in its central part, away from the maximum possible loss $s_{\max }$, by a Weibull distribution of the form

$$
\operatorname{Pr}(\mathrm{S} \geq s) \sim e^{-(s / d)^{c}}
$$

For $\Lambda \rightarrow 1^{-}$, we have $s_{\max } \rightarrow+\infty$ and, for $s \ll s_{\max }$, expression (4) simplifies into a simple exponential function

$$
P_{\Lambda \rightarrow 1^{-}}(S \geq s) \sim e^{-|\ln (\beta)| s / S_{0}}
$$

2. For $\Lambda=1$, the distribution of losses is a simple exponential function since $S_{n}=n S_{0}$ is linear in the number $n$ of stages and the probability of reaching stage $n$ is the exponential $P(n)=\beta^{n}(1-\beta)$. Actually, the expression (6) becomes asymptotical exact as

$$
P_{\Lambda=1}(S \geq s)=(1-\beta) e^{-|\ln (\beta)| s / S_{0}}
$$

3. For $\Lambda>1$, the distribution of losses is of the form,

$$
P_{\Lambda>1}(S \geq s)=\frac{1}{\left(1+\frac{s}{s *}\right)^{c}}, \quad s^{*}:=\frac{S_{0} \Lambda}{\Lambda-1}, \quad c:=\frac{|\ln \beta|}{\ln \Lambda}
$$

which develops to a power law distribution of losses of the form $\operatorname{Pr}(\operatorname{loss} \geq S)=C / S^{\mu}$ with $\mu=c$, when $\Lambda \rightarrow+\infty$.

For $\Lambda \rightarrow 1^{+}$, the tail is still power law and the exponent $\mu$ grows without bound if the probability $\beta$ does not converge to 1 . If both $\Lambda \rightarrow 1^{+}$and $\beta \rightarrow 1^{+}$such that $\Lambda=1-a$, $\beta=1-\rho a$, with $a \rightarrow 0^{+}$and $\rho$ constant, we have $\mu \rightarrow \rho$.

\section{Model calibration}

Figure 4 presents these different regimes and the corresponding parameters calibrated to the PSA curves and to the empirical records. We calibrate the model by using expression (8) because, for both the Farmer curve and empirical records, the solution lies in the region 
$\Lambda>1$. The calibration consists in finding (i) the probability $\beta$ and (ii) the damage factor $\Lambda$ from the ex-ante predictions obtained from the Farmer safety analysis and from the ex-post historical records.

Both distributions are calibrated first by grid search. From the fitted parameters, one hundred distributions are generated and fitted again by grid search. The final parameters of each distribution are the median values of the bootstrapped distributions. The corresponding fits are shown in figure 5 for the Farmer curve (panel A) and for empirical records (panel B). We obtain $\left(\beta_{\text {Farmer }} \approx 0.9 ; \Lambda_{\text {Farmer }} \approx 1.05\right)$ compared with $\left(\beta_{\text {emp. }} \approx 0.95 ; \Lambda_{\text {emp. }} \approx 1.10\right)$. Interpreted within this cascade model, the safety analysis leading to the PSA curve attributes roughly a $90 \%$ probability that an incident having reached a given level of severity may cascade into the next one. To account for the observed distribution of losses, this number needs to be increased by just $5 \%$ to about a $95 \%$ probability of cascading. Thus, an underestimation of just $5 \%$ in the probability $\beta$ for a cascade to continue and in the additional amplifying factor $\Lambda$ has the effect of leading to a significant underestimation of the distribution of losses, in particular for large events. The origin of this sensitivity stems from the proximity of $\beta$ to the critical value 1 , likely due to optimization as occurs in almost any human enterprise associated with the design, modeling, planning and operating of many complex real-world problems [33 35].

\section{CONCLUSIONS}

Development of probabilistic safety assessment (PSA) at level 3, which evaluates the impact of unintended radioactive releases on the public and the environment, has been slowed down and only a few studies exist including a) modifications to enhance operational performance, safety and security enforcing measures, b) improved understanding and modeling of several accident phenomena and potential event sequences, c) advances in PSA technology and other, including simulation techniques and use of huge data. For example, the US NRC has not sponsored development of PSA level 3 since 1990 [4].

In this article, we have presented a structured analysis based on empirical evidence that suggests a poor track record of PSA to reflect adequately the true risks involved in the nuclear industry. In particular, PSA is currently not adapted to identify correctly the likelihood of large catastrophes. The recognition obtained from our study of (i) extreme heavy-tailed 
losses and (ii) the critical nature of cascades during incidents and accidents calls for the integration of the observations and statistics over a large heterogeneous set of incidents into the PSA framework, a procedure that is at present not implemented and seems still far from being considered. If the estimation of total risk is the aim, there is an urgent need to address limitations (despite the acknowledged achievements) and strive to overcome them. Safety analysis should be a never ending process constantly building on past experience, including the evolving full distribution of losses, for the development and the implementation of ever improved measures based on model update [36].

A word of caution should be added, as we use a non-standard database, put together by Sovacool [18], which represents a best effort, but cannot be considered as sufficiently reliable to draw policy recommendations, for instance. However, our method to measure the gap between safety analysis and the size of events at the coarse grained level is a step towards a systematic and continuous comparison between safety analysis and the actual size of events (e.g. in financial loss), at the scale of one facility, or at the scale of a utility (e.g. EDF). Applying our approach to smaller scale studies would also help overcome the problem of the heterogeneity of nuclear power production technologies, which cannot be addressed currently due to the paucity of our data. A utility company which would have gathered data on both (i) distributions generated by PSA, and (ii) loss records, would be in a position to test and refine PSA by using our method.

The method proposed here applies beyond the nuclear industry to many other human innovations and technological developments for which history is a poor guide due to the novelty of the technology and the corresponding lack of history leading to insufficient statistics, in particular for extreme events. Among such difficult unsolved questions, one can cite the impact of cell phones on brain cancer, genetically modified foods and health risks, antibiotic use in cattle and resistant bacterias, biotechnological medicine and health risks, nanotechnology and health risks, Internet and grid technology versus cyber-risks, unequal distribution of gains from capital markets and the (in-)stability of societies, virtual technology and brain development in children and teenagers, human population and technological development and climate change, financial globalization and systemic banking meltdown, nuclear energy and chemical industry versus the risks of release of long-lived toxic elements 
in the biosphere, and so on.

[1] Lee, J.C. and N.J. McCormick, Risk and Safety Analysis of Nuclear Systems, 1st. ed., Wiley (2011)

[2] Kröger, W. and E. Zio, Vulnerable systems, Springer (2011).

[3] Landolt-Börnstein: Numerical Data and Functional Relationships in Science and Technology - New Series, Advanced Materials and Technologies, Nuclear Energy, contributions by Zeynel Alkan, Bertrand Barré, Rudolf Bock, David Campbell, Wolfgang Grätz, Thomas Hamacher, Klaus Heinloth, Dieter H.H. Hoffmann, Ingo Hofmann, William J. Hogan, Wolfgang Kröger, Ernst Kugeler, Kurt Kugeler, Grant B. Logan, Kanetada Nagamine, C.L. Olson, Herwig G. Paretzke, Nathalie Pöppe, 1st edition, Springer, (March 24, 2005).

[4] Borchardt, R. W., Options for proceedings with future Level 3 probabilistic risk assessment activities, SECY-11-0089 (July 7, 2011), available at http://www.nrc.gov/reading-rm/doc-collections/commission/secys/2011/2011-0089scy.pdf

[5] IAEA, Development and Application of Level 1 Probabilistic Safety Assessment for Nuclear Power Plants, IAEA Safety Standards No. SSG-3, Specific Safety Guide, International Atomic Energy Agency, Vienna International Centre, Vienna, Austria (April 2010).

[6] IAEA, Development and Application of Level 2 Probabilistic Safety Assessment for Nuclear Power Plants, IAEA Safety Standards No. SSG-4, Specific Safety Guide, International Atomic Energy Agency, Vienna International Centre, Vienna, Austria (May 2010).

[7] Procedures for Conducting Probabilistic Safety Assessments of Nuclear Power Plants (Level 3), Off-site consequences and estimation of risks to the public Safety Series No 50-P-12, International Atomic Energy Agency, Vienna (1996).

[8] Bonelli, A., O. Mazzantini, M. Sonnenkalb, M. Caputo, J. Matias García, P. Zanocco and M. Gimenez, Station Black-Out Analysis with MELCOR 1.8.6 Code for Atucha 2 Nuclear Power Plant, Science and Technology of Nuclear Installations Volume 2012 (2012), Article ID 620298, 17 pages, doi:10.1155/2012/620298.

[9] Molesh, A., Closing Plenary Speech, PSAM 11 (2012)

[10] Gesellschaft für Reaktorsicherheit (GRS) mbH, Deutsche Risikostudie Kernkraftwerke Eine Untersuchung zu dem durch Störfälle in Kernkraftwerken verursachten Risiko Hrsg,: Der 
Bundesminister für Forschung und Technologie Verlag TÜV Rheinland, Köln (1979).

[11] Marshall, Sir Walter, Design and safety of Sizewell pressurized water reactor, Proc. Roy. Soc. Lond. A 385, 241-251 (1983).

[12] Marshall, E., The Secret Recipe of GE's Reactor Safety Study, Science, 225 (July), 294 (1984).

[13] Speed, T.P., Probabilistic risk assessment for the sizewell B PWR, Radical Statistics Newsletter 29 (Feb.), 4-14 (1984).

[14] Gesellschaft für Reaktorsicherheit (GRS) mbH, German Risk Study, Nuclear Power Plants, Phase B,, GRS-74 (July 1990) ISBN 3-923875-24-X

[15] NUREG-1150, Severe Accident Risks: An Assessment for Five U.S. Nuclear Power Plants Final Summary, Division of Systems Research, Office of Nuclear Regulatory Research, U.S. Nuclear Regulatory Commission, Washington, DC 20555, December 1990.

[16] NEEDS (New Energy Externalities Developments for Sustainability), Project no: 502687, Final report on technical data, costs and life cycle inventories of nuclear power plants, Authors : Claire Lecointe, David Lecarpentier, Vincent Maupu, Denis Le Boulch, Romain Richard (EDF R\&D), with contributions from : Claude Garzenne (EDF R\&D), Till M. Bachmann (EIFER), 21 Dec. .2007.

[17] Bal Raj Sehgal, Light water reactor (LWR) safety, Nuclear Eng. Tech., 38 (8), 697-732 (2006).

[18] Sovacool, B., The costs of failure: A preliminary assessment of major energy accidents, 19072007, Energy Policy, 36, 1802-1820 (2008).

[19] Clery, D. (2011) Current designs address safety problems in Fukushima reactors Science 331, 1506.

[20] Rasmussen, N. C. et al. Reactor safety study. An assessment of accident risks in U. S. commercial nuclear power plants, WASH-1400 (NUREG-75/014), U.S. Nuclear Regulatory Commission, (1975).

[21] Cranmer, K. (2001), Computer Physics Communications 3,198-207.

[22] International Atomic Energy Agency (1996), Defense in Depth in Nuclear Safety.

[23] Peters, K., L. Buzna and D. Helbing (2008) Modelling of cascading effects and efficient response to disaster spreading in complex networks, Int. J. Critical Infrastructures 4, 1/2, 46-62.

[24] Keppenne, C.L., and M. Ghil, Adaptive spectral analysis and prediction of the Southern Oscillation Index, J. Geophys. Res. 97, 20449-20554 (1992). 
[25] Anifrani, J.-C., C. Le Floc'h, D. Sornette and B. Souillard, Universal Log-periodic correction to renormalization group scaling for rupture stress prediction from acoustic emissions, J.Phys.I France 5 (6), 631-638 (1995).

[26] Johansen, A. and D. Sornette, Critical ruptures, Eur. Phys. J. B 18, 163-181 (2000)

[27] Sornette, D., Predictability of catastrophic events: material rupture, earthquakes, turbulence, financial crashes and human birth, Proceedings of the National Academy of Sciences USA 99 (Suppl. 1), 2522-2529 (2002).

[28] Ghil, M., I. Zaliapin and B. Coluzzi, Boolean delay equations: A simple way of looking at complex systems, Physica D 237, 2967-2986 (2008).

[29] Balcerak, E., Pairing simple and complex models could improve predictions, Eos, Transactions American Geophysical Union 93 (8), 86-87 (2012).

[30] Kesten H., Random difference equations and renewal theory for products of random matrices, Acta Math. 131, 207-248 (1973).

[31] Sornette, D. and R. Cont, Convergent multiplicative processes repelled from zero: power laws and truncated power laws, J. Phys. I France 7, 431-444 (1997).

[32] Sornette, D., Multiplicative processes and power laws, Phys. Rev. E 57 (N4), 4811-4813 (1998).

[33] Sornette, D., Critical phase transitions made self-organized : a dynamical system feedback mechanism for Self-Organized Criticality, J.Phys. I France 2, 2065-2073 (1992).

[34] Carlson, J.M. and J. Doyle, Complexity and robustness, Proc. Natl. Acad. Sci. USA 99 (Suppl 1), 2538-2545 (2002).

[35] Sornette, D., Critical Phenomena in Natural Sciences 2nd ed., Springer Series in Synergetics, Heidelberg (2004).

[36] Sornette, D., A. B. Davis, K. Ide, K. R. Vixie, V. Pisarenko, and J. R. Kamm, Algorithm for Model Validation: Theory and Applications, Proc. Nat. Acad. Sci. USA 104 (16), 6562-6567 (2007). 


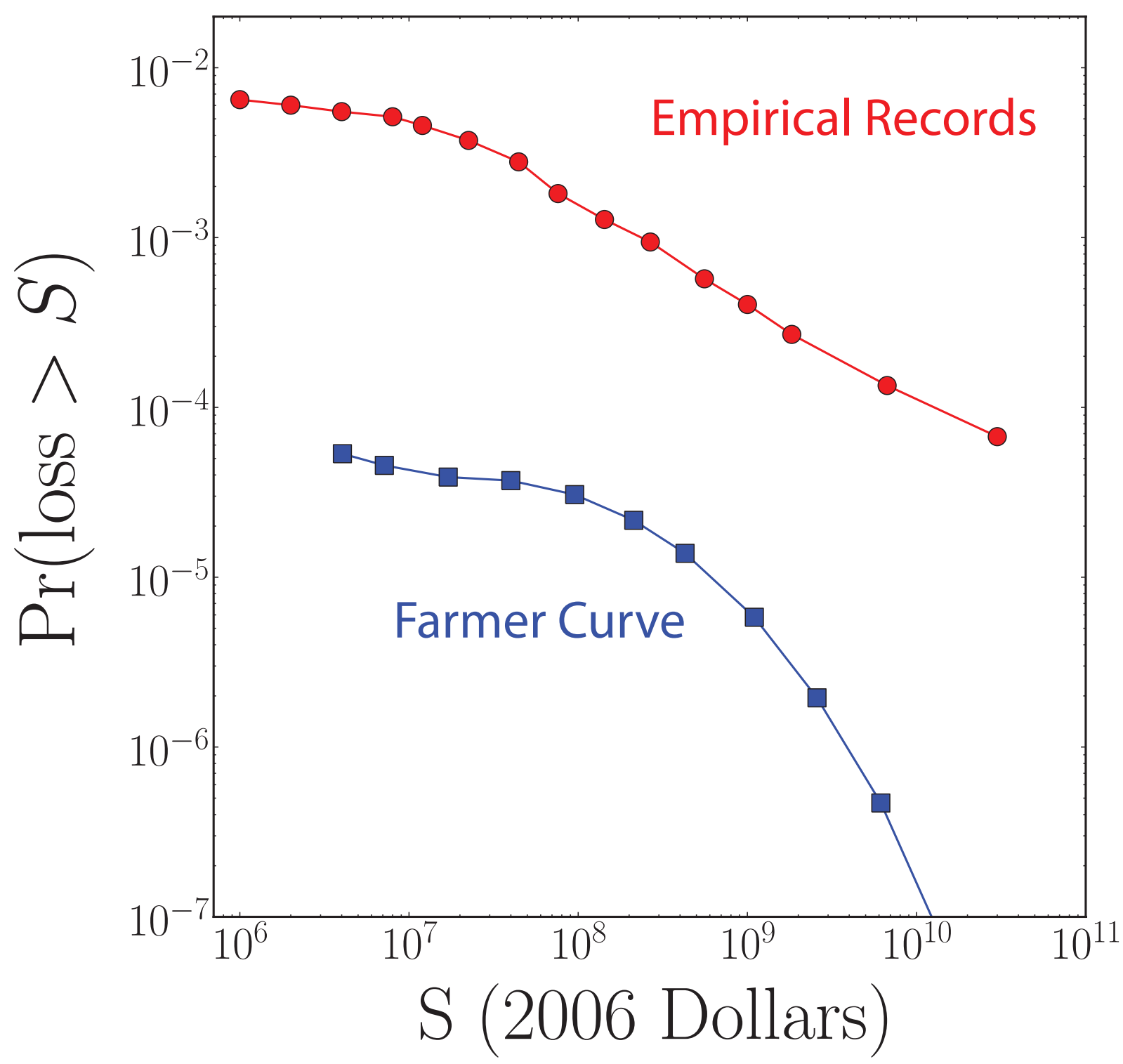

FIG. 1: (color online) Distribution of losses $S$ normalized for 1 nuclear plant $\times$ year, obtained (i) from probabilistic safety assessment (PSA) and (ii) from empirical records [18]. We refer to the PSA distribution as the "Farmer curve" for historical reason, even if we use the more recent Rasmussen curve of the 1970s [17, 20] for 100 reactors, which is basically confirmed by others (German Risk Study, figures 8-13, 14) or NUREG [15]. Safety analysis largely underestimates the losses due to nuclear incidents. The difference is striking in the tail of the distribution: the distribution obtained from the PSA method vanishes fast beyond $\$ 1$ billion damage while empirical records exhibit a power law tail with exponent $\mu=0.7 \pm 0.1$ with no apparent cut-off. 
(A)

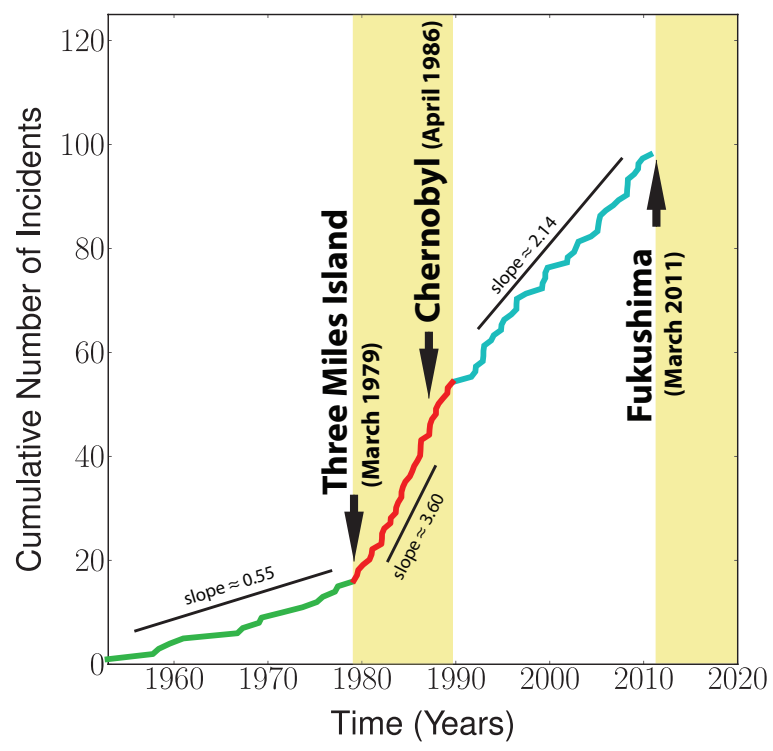

(B)

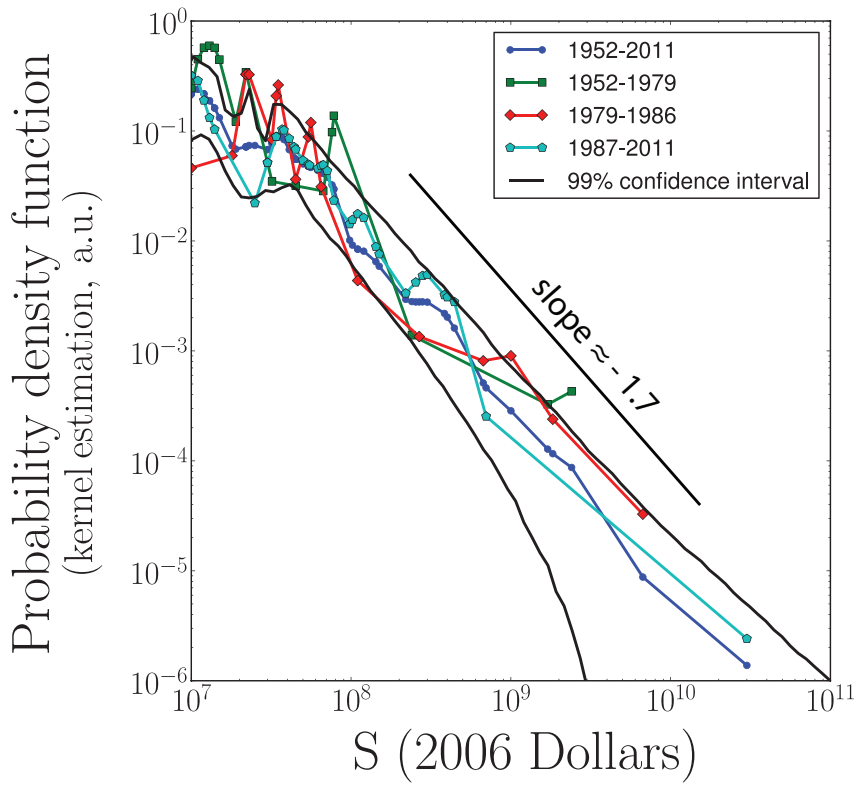

FIG. 2: (color online) (A) Cumulative number of civil nuclear incidents over time since 1957. Three regimes can be distinguished: (i) the burgeoning nuclear industry with small but quickly increasing installed capacity with an average rate of 0.55 incidents per year; (ii) from the time of the Three Mile Island accident to the Chernobyl accident, a rate of 3.6 incidents per year; (iii) the post-Chernobyl era is characterized by a rate of slightly above 2 incidents per year. (B) Test for stability of the empirical complementary cumulative distribution function (CCDF) over three distinct time intervals of nuclear power industry history and over the whole period, using adaptive kernel density estimators [21]. 

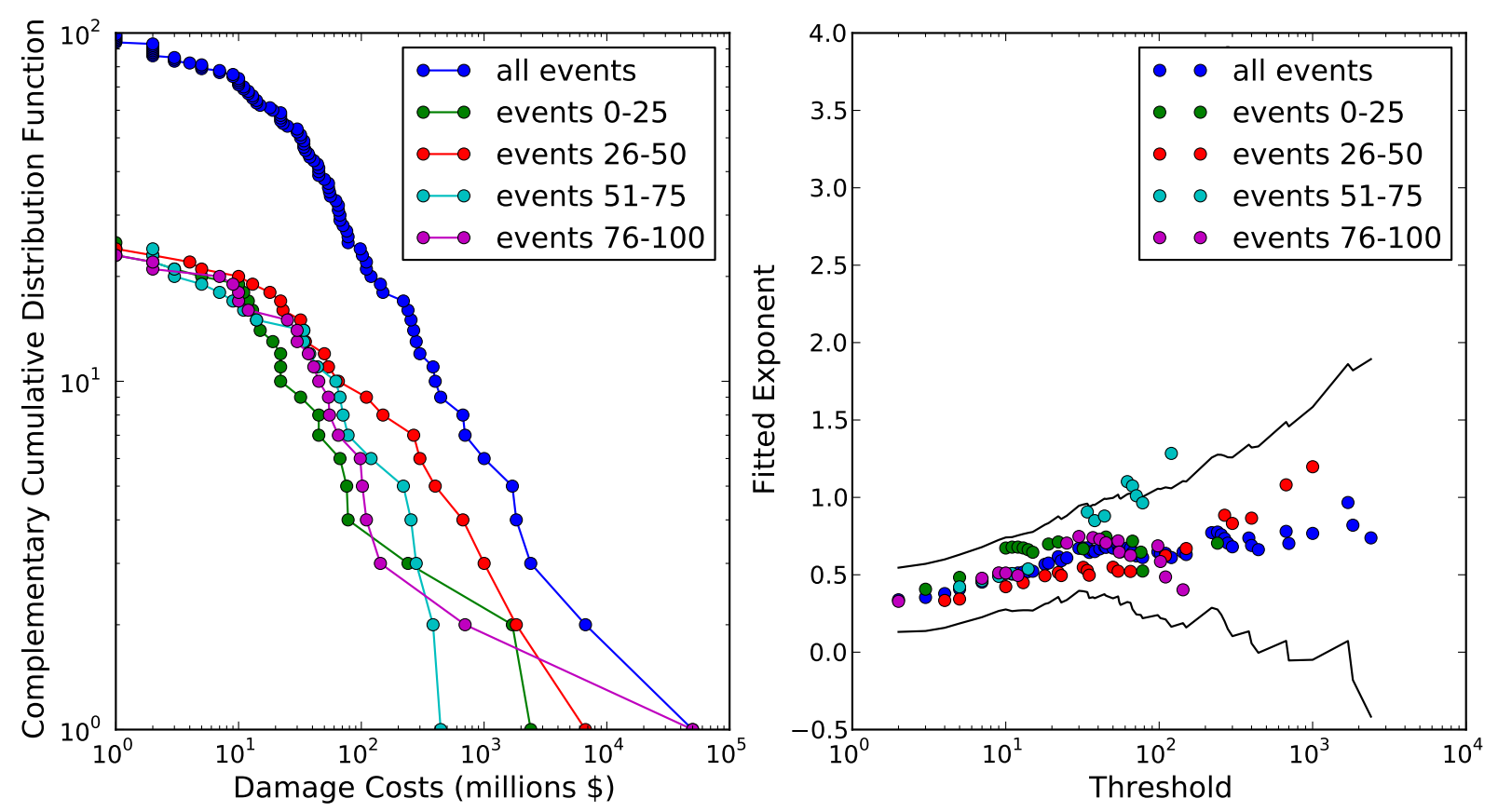

FIG. 3: (color online) Left panel shows the survival distribution functions of loss per nuclear incident, broken down in periods of times from 1957 to 2011 with 25 events in each time interval. The tails of the distributions for the five time periods have the same power law structure, as confirmed by the maximum likelihood estimates (MLE) of the tail exponent $\mu$ for each distribution shown in the right panel. The MLE are calculated for each of the five distributions by varying the lower threshold in units of million US\$ (abscissa), i.e. by taking into account only the losses larger than the threshold value given by the abscissa in the right panel. This allows us probing the stability of the power law tail. One can observe the exponents clustering around the value $\mu=0.7$ for thresholds larger than $\$ 30$ million. The two continuous lines indicate the $95 \%$ confidence interval for the MLE of the exponent $\mu$. 


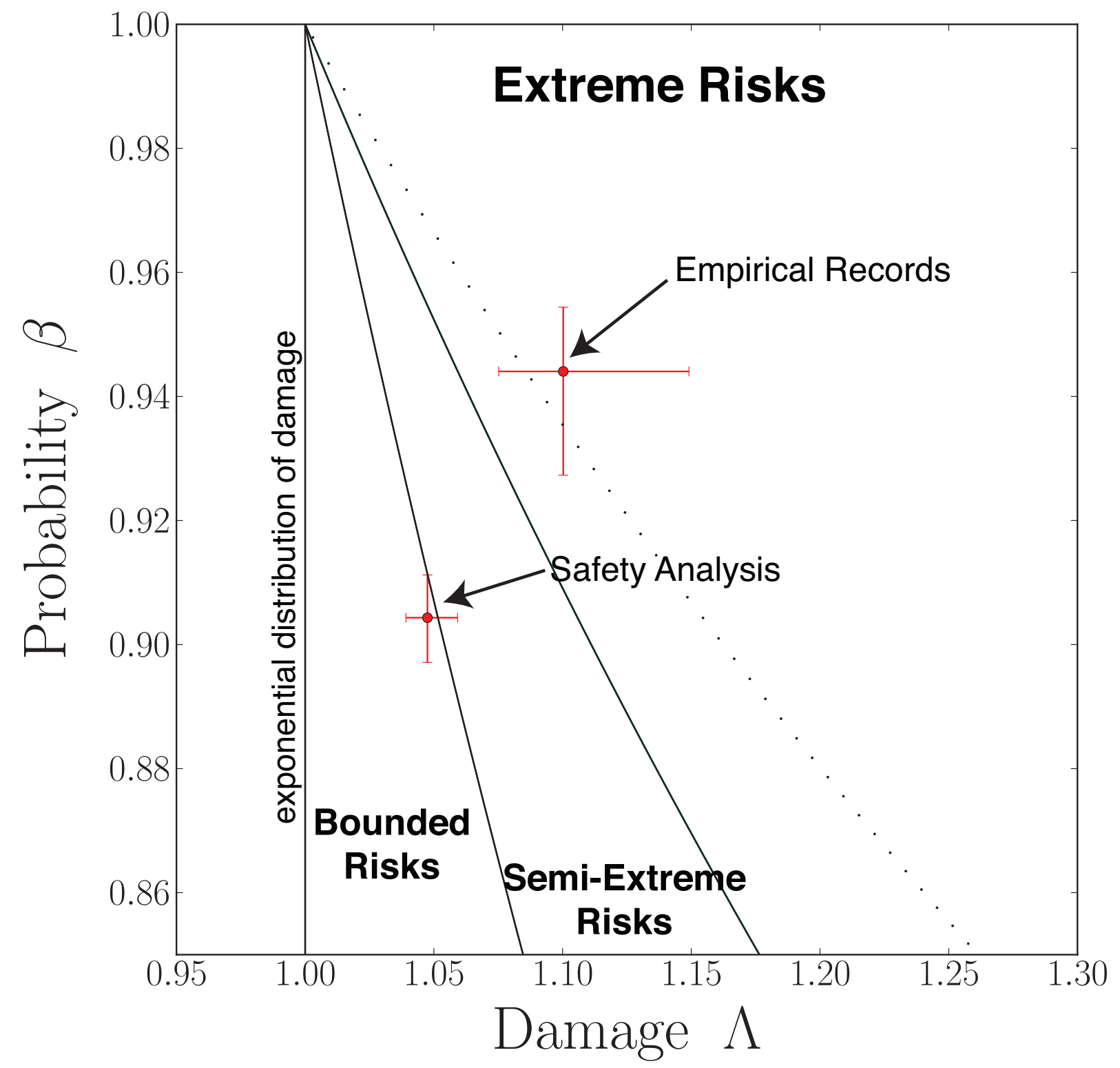

FIG. 4: (color online) Phase diagram showing the three regions of fat-tail risks predicted by the cascade model: (i) bounded risks with mean and variance defined (exponent $2<\mu$ ), (ii) semiextreme risks with only mean defined and variance undefined (exponent $1<\mu \leq 2$ ) and (iii) extreme risks with unbounded mean and variance (exponent $\mu \leq 1$ ). Empirical records clearly identify nuclear accidents as extreme risks (upper right red point with confidence intervals), whereas safety analysis predicts that damage following nuclear incidents is fat-tailed yet bounded (lower left). 
(A)

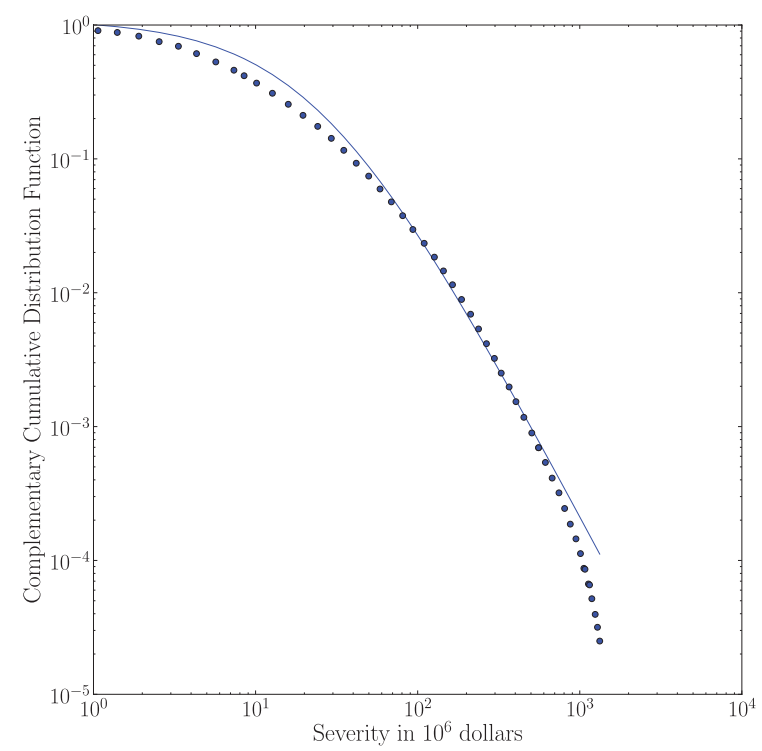

(B)

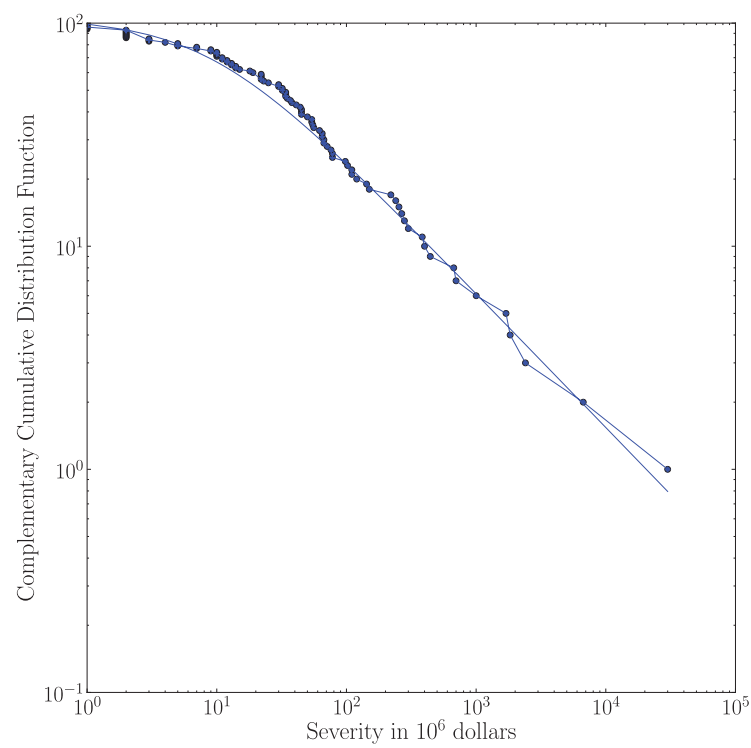

FIG. 5: Calibration of the accident propagation cascade model with expression (8). For the safety analysis (Farmer curve in panel (A)), we find that the probability of propagation is $\beta=0.9$ (lower bound $\beta_{05}=0.9$, upper bound $\beta_{95}=0.91$ at $95 \%$ confidence intervals), and the damage factor is $\Lambda=1.05$ (lower bound $\Lambda_{05}=1.04$, upper bound $\Lambda_{95}=1.06$, at $95 \%$ confidence interval) for $S_{\min }=10^{7}$. Panel (B) shows the best fit of expression (8) to the real data is found for $\beta=0.95$ (lower bound $\Lambda_{05}=0.94$, upper bound $\Lambda_{95}=0.96$ and $\Lambda=1.10$ (lower bound $\Lambda_{05}=1.08$, upper bound $\left.\Lambda_{95}=1.15\right)$. 